\title{
Plasmon resonance sensitivity in fine metal particle
}

\author{
N.I. Grigorchuk \\ Bogolyubov Institute for Theoretical Physics, National Academy of Sciences of Ukraine, \\ 14-b Metrologichna St., 03680 Kyiv, Ukraine
}

Received September 26, 2013, in final form November 4, 2013

\begin{abstract}
The sensitivity of the wavelength position of surface plasmon resonances in prolate and oblate fine metal particles to the refractive index of an embedding solution, a particle shape, and electron temperature is studied theoretically in the framework of different analytical methods. All calculations are illustrated on the single potassium nanoparticle as an example.
\end{abstract}

Key words: sensitivity, surface plasmon resonance, fine metal particles

PACS: 78.67.-n, 65.80.-g, 73.23.-b, 68.49.Jk, 52.25.0s

The wavelength, intensity, and shift of the surface plasmon resonance (SPR) [1] of fine metal particles (MPs) are sensitive to changes in different physical parameters. The possibility to measure the optical effects with the refractive index variation has been often used to detect changes in the surrounding medium for chemical sensing or for probing the dynamics of biological molecules [2]. An important group of optical sensors is based on exploiting SPR to detect small refractive index changes in close proximity to the sensing surface of MPs [3-11] and are associated with a biomolecule sensing [8, 9]. Several authors have demonstrated, on the example of noble nanoparticles, how the SPR position depends on the environment and the morphology of nanoparticles [3-5, 7]. Much less attention has been paid to the study of sensitivity modes of a spheroidal MP with a small effect of a core polarization, where the contribution of the bound electrons can be neglected (e.g., $\mathrm{K}, \mathrm{Na}, \mathrm{Be}, \mathrm{Al}$, etc).

In this Letter, using the new approach specifying the nanoparticle shape, we propose the expressions for calculation of the plasmonic sensitivity to the changes in the main factors that significantly affect the position of the SPR modes on the example of potassium nanoparticle.

1. The MPs considered are small in size relative to the wavelength of light. If we change the refractive index of the embedding medium $n$ by a definite value, $\delta n$, the sensitivity can be expressed in frequency units as $S_{j, \omega}(n)=\partial \omega_{j, \text { res }} / \partial n$. The resonance frequency (or the corresponding wavelength) for nonspherical MP is given by [4]

$$
\begin{gathered}
\omega_{j, \text { res }}=\omega_{\mathrm{pl}} / \sqrt{\varepsilon_{\infty}+\left(1 / L_{j}-1\right) n^{2}}, \\
\lambda_{j, \text { res }}=\lambda_{\mathrm{pl}} \sqrt{\varepsilon_{\infty}+\left(1 / L_{j}-1\right) n^{2}},
\end{gathered}
$$

where the Drude model with $\operatorname{Re}(\varepsilon) \simeq \varepsilon_{\infty}-\omega_{\mathrm{pl}}^{2} / \omega^{2}$ is used, $\omega_{\mathrm{pl}}$ is the plasma frequency, $\varepsilon_{\infty}$ is the high frequency dielectric constant, and $L_{j}$ refers to the Osborn's demagnetizing factors [12] in $j$-th direction.

We have restricted ourselves to the MPs with a spheroidal shape. Then, variables $L_{\|}$and $L_{\perp}$ will play the roles of longitudinal (directed along the revolution axis of a spheroid) and transverse (directed across this axis) components of the $L$ factor. Since Osborn's factors are somewhat cumbersome to be used directly, they can be changed with a high accuracy by the following relations

$$
L_{\|}=\frac{1}{\left(1+R_{\|} / R_{\perp}\right)^{z}}, \quad L_{\perp}=\frac{1}{2}\left(1-L_{\|}\right),
$$

where $z=\log 3 / \log 2, R_{\|}$and $R_{\perp}$ are the spheroid semiaxes directed along and across the revolution axis of a spheroid, correspondingly. 
Equation (2) describes the change in the SPR wavelength when the factors $L_{j}$ or refractive index are changed. Figure 1 depicts the result of calculations for a rodlike potassium nanoparticle. One can see that the SPR peak wavelength $\lambda_{\text {res }}$ (normalized by plasma wavelength $\lambda_{\mathrm{pl}}=2822 \AA$ for K) linearly depends on $n$, and is shifted to the red spectral side as $n$ is increased. Physically, this implies that the Coulomb attractive force between an electronic cloud and a positive metal lattice is weakened with $n$ and the energy of SPR is reduced. The linear increase of SPR peak with $n$ retains for different values of the aspect ratio $R_{\|} / R_{\perp}(\equiv a / b=x)$. Only the line slope changes: it enhances for MPs with larger aspect ratios. From the slope of the linear fit, the refractive index sensitivity $\partial \lambda_{\text {res }} / \partial n$ was calculated to be 256 $\mathrm{nm}$ per refractive index unit (RIU) for $S_{\perp}$ and $380 \mathrm{~nm}$ per RIU for $S_{\|}$, with "golden" $x=1.618$, or to be $205 \mathrm{~nm} / \mathrm{RIU}$ for $S_{\perp}$ and $2100 \mathrm{~nm} / \mathrm{RIU}$ for $S_{\|}$, with $x=16.18$. A tenfold increase of the potassium particle aspect ratio enhances the $S_{\|}$-sensitivity to the changes in the refractive index roughly 5 times. Higher spheroid aspect ratio supports both the $\|$-SPR mode at a longer wavelength and the $\perp$-mode at a rather shorter wavelength. One can see that the \|-plasmon mode is much more sensitive to the change in the refractive index than the transverse mode.

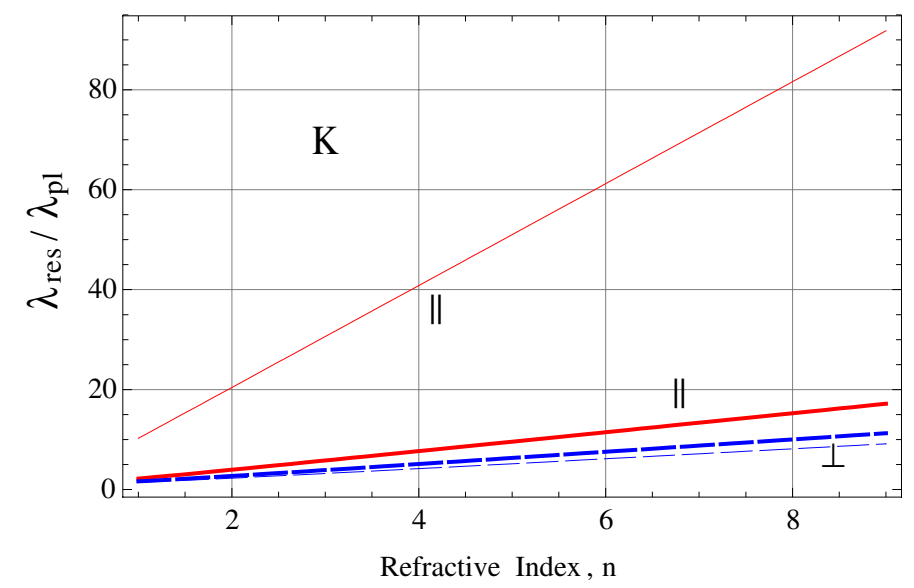

Figure 1. (Color online) SPR peak wavelength modes $(\|$ and $\perp$ ) position as a function of medium refractive index for prolate potassium nanoparticle with different aspect ratios: $R_{\|} / R_{\perp}=1.618$ (thick lines) and = 16.18 (thin lines).

2. By an analogy with the previous case, the sensitivity to the changes in the aspect ratio of a spheroidal nanoparticle $x$ can be expressed in frequency units as $S_{j, \omega}(x)=\partial \omega_{j, \text { res }} / \partial x$. Using equations (1) and (2) in the determination of sensitivity, one obtains

or

$$
S_{j, \omega}(x)=\frac{n \omega_{\mathrm{pl}}\left[1 / L_{j}(x)-1\right]}{\left\{\varepsilon_{\infty}+n^{2}\left[1 / L_{j}(x)-1\right]\right\}^{3 / 2}},
$$

$$
S_{j, \lambda}(x)=\frac{n \lambda_{\mathrm{pl}}\left[1 / L_{j}(x)-1\right]}{\sqrt{\varepsilon_{\infty}+n^{2}\left[1 / L_{j}(x)-1\right]}} .
$$

We can see that the sensitivity of the SPR to the medium can be enhanced by tuning the morphology of the nanoparticle [3-5, 7]. The shape factor $L_{j}(x)$, which governs the geometric tunability of the SPR, also determines the plasmon sensitivity of nanoparticles. It is easy to find those values of $L_{j}$ for which the sensitivity $S_{j}(x)$ becomes maximal. They can be expressed in the wavelength or frequency units by means of expressions

$$
L_{\|, \lambda}=\frac{n^{2}}{n^{2}-2 \varepsilon_{\infty}}, \quad L_{\|, \omega}=\frac{n^{2}}{n^{2}+2 \varepsilon_{\infty}}
$$

for a maximal longitudinal component of the $S_{\|}$, or

$$
L_{\perp, \omega}=\frac{2 \varepsilon_{\infty}-n^{2}}{2 \varepsilon_{\infty}+n^{2}}
$$


for a maximal transverse component of the $S_{\perp}$

Only a few MPs and dielectric media conform to the first of the two equation (6). For this reason, we propose somewhat different approach. The derivative $\partial \lambda_{\text {res }} / \partial n$ can be represented as a derivative of a composite function

$$
\frac{\partial \lambda_{\text {res }}}{\partial n}=\frac{\partial \lambda_{\text {res }}}{\partial \varepsilon^{\prime}} \frac{\partial \varepsilon^{\prime}}{\partial n}
$$

where $\varepsilon^{\prime}$ is the real part of a particle dielectric function. In the high frequency limit of dielectric function, $\lambda_{\text {res }}$ is

$$
\lambda_{\text {res }}=\lambda_{\mathrm{pl}} \sqrt{\varepsilon_{\infty}-\varepsilon^{\prime}}
$$

From the plasmon resonance condition

$$
\varepsilon^{\prime}=-\left(1 / L_{j}-1\right) n^{2}
$$

and finally, we get

$$
\frac{\partial \lambda_{j, \mathrm{res}}}{\partial n}=\frac{\lambda_{\mathrm{pl}}^{2}}{\lambda_{j, \mathrm{res}}}\left(1 / L_{j}-1\right) n .
$$

In the case of MP having a spherical form, the factor $L_{j}$ should be put equal to $1 / 3$ in all the above equations.

3. Some experimental studies [3-5, 10, 13-16] were carried out on metal nanoparticles with different geometry to find the best nanoparticle configuration to enhance the sensitivity of the SPR response. Let us theoretically find the best geometrical factors $L_{j}$ to enhance the sensitivity of the SPR response for MPs with a spheroidal shape. To define the spheroid shape which provides the maximal sensitivity, one can use the following relations:

$$
\begin{aligned}
& \left(R_{\|} / R_{\perp}\right)_{\text {long }}=\left(L_{\|, \omega}\right)^{-1 / z}-1, \\
& \left(R_{\|} / R_{\perp}\right)_{\text {trans }}=\left(L_{\perp, \omega}\right)^{-1 / z}-1,
\end{aligned}
$$

for longitudinal and transverse components, correspondingly.

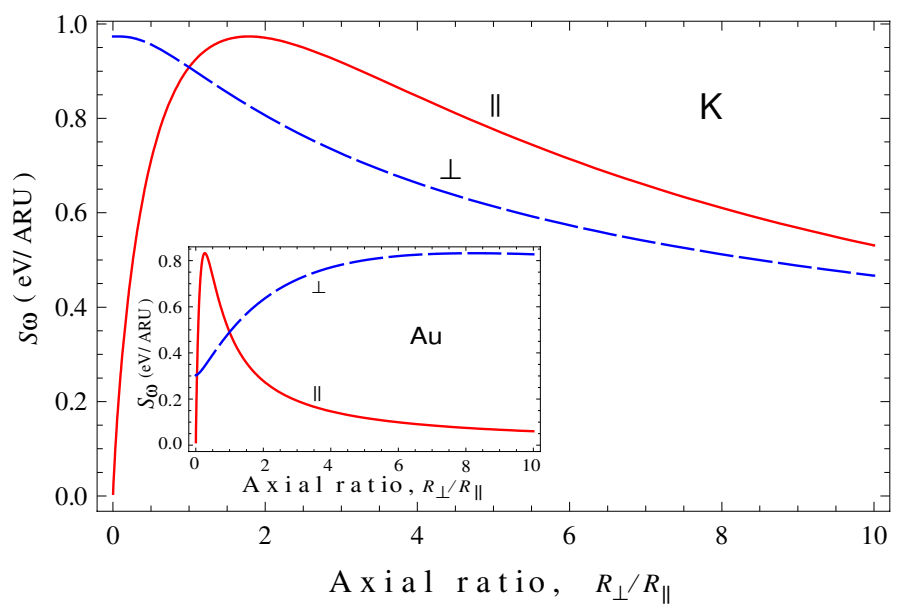

Figure 2. (Color online) Dependence of the sensitivity of the plasmon resonance components (longitudinal - solid line and transverse - dashed line) vs semiaxial ratio for spheroidal potassium nanoparticle embedded in the $\mathrm{SiO}_{2}$ matrix $(n=1.56)$. The inset shows the same dependence for Au nanoparticle embedded in the water.

For instance, if we take the Au nanoparticle with $\varepsilon_{\infty}=9.84$ [10] embedded in water $(n=1.33)$, then from the second equation (6) and equation (7), we obtain $L_{\omega} \simeq 0.082$ for $\|$ and $L_{\omega} \simeq 0.835$ for $\perp$ components, which enable us to get from equations (12) and (13) the maxima at $\left(R_{\|} / R_{\perp}\right)_{\text {long }}=3.76$ and at $\left(R_{\|} / R_{\perp}\right)_{\text {trans }}=0.12$. 
Figure 2 shows the dependence of plasmon sensitivity components on the axial ratio (in axial ratio units (ARU)) calculated for the potassium nanoparticle with $\varepsilon_{\infty}=1.24$ [17]. We have found that the maximal sensitivity can be realized at the axial ratio $\left(R_{\perp} / R_{\|}\right)_{\text {long }} \simeq 2$ for $S_{\|}$, and at $\left(R_{\perp} / R_{\|}\right)_{\text {trans }} \simeq 0$ for $S_{\perp}$. The inset in figure 2 displays the same dependence $S_{E}(x)$ for Au nanoparticle. Maximal sensitivities are attained for Au at $\left(R_{\perp} / R_{\|}\right)_{\text {long }} \simeq 0.26$ and $\left(R_{\perp} / R_{\|}\right)_{\text {trans }} \simeq 8.5$ for $S_{\|}$and $S_{\perp}$, correspondingly. Such quantities of maximal sensitivities for potassium and Au nanoparticles can be obtained from equations (12) and 13) as well. The data in figure 2 for prolate and oblate potassium and Au nanoparticles display a clear evidence of the spectral shift of the both components $S_{\|}$and $S_{\perp}$ as a function of spheroidal axial ratios. Such a behavior of both $S(x)$ components is far from the linear one predicted, e.g., in [3] for the single Au nanorod.

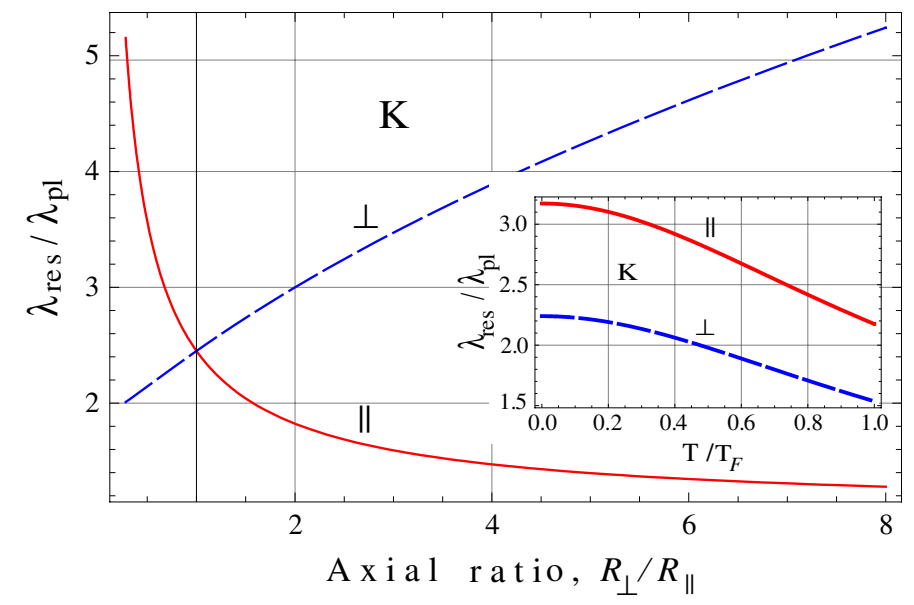

Figure 3. (Color online) The SPR peak wavelength components ( $\|$ and $\perp$ ) as a function of the spheroid axial ratio for potassium nanoparticle embedded in the $\mathrm{SiO}_{2}$ matrix. The inset shows $\lambda_{\text {res }}(T)$ for potassium nanoparticle (with $x=1.62$ ) embedded in the $\mathrm{SiO}_{2}$ matrix. For potassium the Fermi temperature is $T_{\mathrm{F}}=2.46 \times 10^{4} \mathrm{~K}$ [17] and electron concentration is $n_{0}=1.4 \times 10^{22}$ [17].

In figure 3 the dependence of the ratio $\lambda_{\text {res }} / \lambda_{\text {pl }}$ versus spheroid axial ratio is depicted. As it is seen in this figure, when the aspect ratio for rodlike potassium nanoparticle increases, the \|-component of the peak of the SPR wavelength $\lambda_{\text {res }}$ exhibits a blue shift, whereas its $\perp$-component exhibits a red shift. For oblate potassium nanoparticle, vice versa, with an increase of the semiaxial ratio $R_{\perp} / R_{\|}$, the $\|$-component of the SPR wavelength shifts to the red side, whereas its $\perp$-component shifts to the blue side. From the slope of the linear dependence of $\lambda_{\perp, \text { res }}$ on the axial ratio, the shape sensitivity $\partial \lambda_{\text {res }} / \partial x$ becomes roughly as high as $84 \mathrm{~nm} / \mathrm{ARU}$ for $S_{\perp}$.

4. To characterize the thermal stability of the photonic crystal devices, the study of their thermal sensitivity is very important [18-20]. It can be expressed in wavelength units as $S_{j, \lambda}(T)=\partial \lambda_{j, \text { res }} / \partial T$. The temperature dependence of $\lambda_{\text {res }}(T)$ comes from the $T$-dependence of the electron concentration inside the MP [21]

$$
n_{\mathrm{e}}(T)=n_{0}\left[1+\frac{\pi^{2}}{8}\left(\frac{k_{\mathrm{B}} T}{\mu_{0}}\right)^{2}\right]
$$

where $\mu_{0}=\mu(T=0)$ is chemical potential at zero temperature and $n_{0}=\left(2 m \mu_{0}\right)^{3 / 2} / 3 \pi^{2} \hbar^{3}$. Combining these equations with $\lambda_{\mathrm{pl}}(T)=2 \pi c / \sqrt{4 \pi n_{\mathrm{e}}(T) e^{2} / m}$ and equation (2), we obtain

$$
\lambda_{j, \mathrm{res}}(T) \simeq \lambda_{\mathrm{pl}}(0) \sqrt{\frac{\varepsilon_{\infty}+\left(1 / L_{j}-1\right) n^{2}}{1+\left(\pi^{2} / 8\right)\left(k_{\mathrm{B}} T / \mu_{0}\right)^{2}}} .
$$


Therefore,

$$
S_{j, \lambda}(T) \simeq \lambda_{\mathrm{pl}}(0) \frac{\pi^{2}}{8} \frac{\sqrt{\varepsilon_{\infty}+\left(1 / L_{j}-1\right) n^{2}}}{\left[1+\left(\pi^{2} / 8\right)\left(k_{\mathrm{B}} T / \mu_{0}\right)^{2}\right]^{3 / 2}}\left(\frac{k_{\mathrm{B}}}{\mu_{0}}\right)^{2} T .
$$

Equations (15) and (16) allow one to estimate the shift of SPR wavelength position with temperature and the temperature sensitivity for any ellipsoidal MPs. It is easy to see from equation (15) and from the inset in figure 3 that the value of $\lambda_{\text {res }}$ is shifted with $T$ to the shorter wavelength side, which agrees well with the results of works [19, 20].

In summary, we propose the formulae that enable one to evaluate the plasmonic sensitivity to the changes in the main factors considerably affected the SPR mode position in MPs. For prolate (oblate) potassium and $\mathrm{Au}$ nanoparticles, there was found a nonlinear behavior of both sensitivity components $\left(S_{\|}\right.$and $S_{\perp}$ ) as a function of an axial ratio. The effect of the temperature variation on the plasmon sensitivity was demonstrated on the example of a potassium nanoparticle.

\section{References}

1. Mayergoyz I.D., Plasmon Resonances in Nanoparticles, World Scientific, Singapore, 2013.

2. Mayer K.M., Lee S., Liao H., Rostro B.C., Fuentes A, Scully P.T., Nehl C.L., Hafner J.H., ACS Nano, 2008, 2, 687; doi $10.1021 / \mathrm{nn} 7003734$

3. Lee K.-S., El-Sayed M.A., J. Phys. Chem. B, 2006, 110, 19220; doi 10.1021/jp062536y

4. Becker J., Trügler A., Jakab A., Hohenester U., Sönnichsen C., Plasmonics, 2010, 5, 161; doi 10.1007/s11468-010-9130-2.

5. Miller M.M., Lazarides A.A., J. Opt. A: Pure Appl. Opt., 2006, 8, S239; doi 10.1088/1464-4258/8/4/S26

6. Tetz K.A., Pang L., Fainman Y., Optics Lett., 2006, 31, 1528; doi $10.1364 /$ OL.31.001528

7. Mock J.J., Smith D.R., Schultz S., Nano Lett., 2003, 3, 485; doi 10.1021/nl0340475

8. Haes A.J., Zou S.L., Schatz G.C., Van Duyne R.P., J. Phys. Chem. B, 2004, 108, 6961; doi 10.1021/jp036261n

9. Otte M.A., Sepúlveda B., Ni W., Juste J.P., Liz-Marzán L.M., Lechuga L.M., ACS Nano, 2010, 4, 349; doi $10.1021 / \mathrm{nn} 901024 \mathrm{e}$.

10. Cao M., Wang M., Gu N., J. Phys. Chem. C, 2009, 113, 1217; doi 10.1021/jp808000x

11. Grigorchuk N.I., J. Phys. Chem. C, 2012, 116, 23704; doi 10.1021/jp3067074

12. Osborn J.A., Phys. Rev., 1945, 67, 351; doi 10.1103/PhysRev.67.351

13. Karakouz T., Holder D., Goomanovsky M., Vaskevich A., Rubinstein I., Chem. Mater., 2009, 21, 5875; doi $10.1021 / \mathrm{cm} 902676 \mathrm{~d}$

14. Sau T.K., Rogach A.L., Jäckel F., Klar T.A., Feldmann J., Adv. Mater., 2010, 22, 1805; doi 10.1002/adma.200902557

15. Chen H., Kou X., Yang Z., Ni W., Wang J., Langmuir, 2008, 24, 5233; doi 10.1021/la800305j]

16. Kelly K.L., Coronado E., Zhao L.L., Schatz G.C., J. Phys. Chem. B, 2003, 107, 668; doi 10.1021/jp026731y

17. Kittel Ch., Introduction to Solid State Physics, Wiley, New York, 1986.

18. Florous N.J., Saitoh K., Koshiba M., IEEE Trans. Nanotechnol., 2007, 6, 549; doi 10.1109/TNANO.2007.901187

19. Dalacu D., Martinu L., Appl. Phys. Lett., 2000, 77, 4283; doi 10.1063/1.1334362.

20. Yeshchenko O.A., Bondarchuk I.S., Alexeenko A.A., Kotko A.V., Functional Materials, 2013, 20, 357.

21. Levich V.G., Course of Theoretical Physics, Vol.1, Nauka, Moskow, 1969 (in Russian). 


\section{Чутливість плазмонних резонансів у малих металевих \\ частинках}

\section{M.I. Григорчук}

Інститут теоретичної фізики ім. М.М. Боголюбова НАН України, вул. Метрологічна, 14-6, 03680 Київ, Україна

В рамках різних аналітичних методів теоретично вивчається чутливість положення довжин хвилі резонансів поверхневого плазмона у витягнутих і сплюснутих малих металевих частинках до показника заломлення оточуючого розчину, форми самої частинки та електронної температури. Всі обчислення проілюстровані на прикладі окремої наночастинки калію.

Ключові слова: чутливість, поверхневий плазмонний резонанс, малі металеві частинки 